\title{
Status and Development of Surface Alloying by Electron Beam
}

\author{
Junwei Duan ${ }^{1}$, Yiping Huang ${ }^{1}$, Ruibin zhang ${ }^{2}$, \\ Li Wan ${ }^{1}$, Hang Li ${ }^{1}$, Juan Liu ${ }^{1}$ \\ ${ }^{1}$ Department of Electrical and Mechanical Engineering, Guilin University of Electronic Technology, \\ Guilin, 541004, China \\ ${ }^{2}$ Department of Automobile Engineering, Guilin University of Aerospace Technology, Guilin \\ Guangxi, 541004, China
}

Keywords: Surface alloying by electron beam, surface modification, intense pulsed electron beam.

\begin{abstract}
In The technology of surface alloying by electron beam had been developed as a new surface treatment in recent decades. Based on the introduction of the principle of electron beam, focuses on the research status of the surface alloying by electron beam, and a brief introduction of the Surface alloying by Intense pulsed electron beam, pointed out as a method of surface modification the Surface alloying by electron beam will occupy a strong competitive advantage irreplaceable position.
\end{abstract}

\section{Introduction}

With the progress of science and technology development and equipment manufacturing industry, the surface properties of materials have become increasingly demanding. In many cases, the performance and quality of components, parts mainly depends on the performance and quality of the material surface, so by improving the surface of morphology, chemical composition, organizational structure in order to improve the material properties of the surface modification technology has been developing rapidly in recent years. Because of their high energy-density, electric conversion efficiency, simple control and high precision, making the research and application of high-energy electron beam surface treatment technology has been widespread concern [1-5].

This paper outlines the principle of electron beam surface alloying, recent progress electron beam surface alloying on briefly described, and describes the surface alloying by pulsed electron beam.

\section{Problems Principle of the electron beam}

The technology of surface alloying by High-energy electron beam has been developed in recent years [6]. Using an electron beam as a heat source acting on the work piece surface is the rapid melting of the material forming the surface layer of the modified layer with special physical and chemical properties of the modified layer can serve to improve the surface hardness, abrasion resistance corrosion resistance and other properties, to compensate for the lack of surface properties.

Electron beam surface alloying has two main characteristics: First, as needed, to select the alloy powder having the desired properties to achieve surface alloying to make the material surface to achieve the desired performance requirements; the second is to be selected according to certain specific needs strengthening parts of surface alloying to achieve the effect of topical treatment [7].

\section{Research evolution of the surface alloying by electron beam}

Yiping Huang [8] considers the influence of the change with temperature for thermal properties; by finite element method Analyze the process of plasma spary. He found that the droplet deposition process of cooling the temperature distribution is uneven, the middle part of the droplet surface 
temperature to drop rapidly, and the rate of decline is easing; local temperature of the substrate near the interface rapid rise, then slow decline after reaching the maximum. It can be seen from Figure 1: With the preheating temperature of substrate rise, the maximum stress decrease.

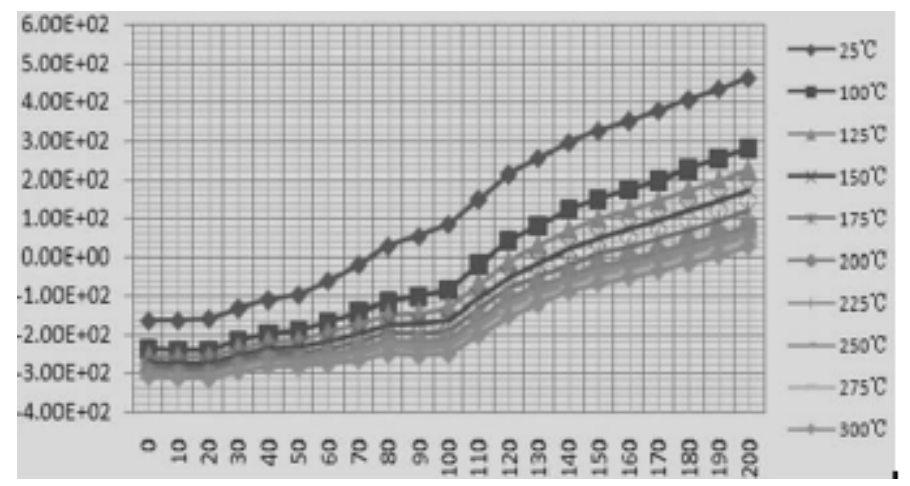

Fig. 1 Substrate temperature corresponds to the radial stress

Xiaoxia Zhang [9] used the method of finite difference and method of finite element, the establishment of a two-dimensional axisymmetric model, dynamic temperature field and stress field titanium alloys under strong effect of pulsed electron beam generated by numerical simulation.

Wulin Song [10] was studied cladding under vacuum that laser cladding under vacuum to avoid intrusion of harmful gases in the atmosphere of the pool, the vacuum can effectively improve the quality of the cladding. Cladding under vacuum reduces the porosity and other microscopic defects and improve its anti-cracking ability.

Benfeng Lu [11] conducted a surface alloying for steel respectively use laser and electron beam. Cladding obtained by electron beam has high carbon content, so does microhardness. Organization uniformity of cladding layer by electron beam is better, Under the conditions of the low stress abrasion, cladding layer by electron beam have better abrasion resistance.

Crack is often an important factor in causing the degradation and failure of the coating of cladding. Studies have shown that the cracks of the electron beam cladding and composite of coatings have a relationship, process parameters have a great impact on the cracks after cladding [12-13].

Jun He [14] tries to surface alloying by electron beam experiments in niobium alloy surface. The results show that: the tearing tendency will tend to increase with the decrease of after the first with the increase of the electron beam, focus current and sweep speed. By controlling the parameters of the surface alloying by electron beam can be in a suitable range of heat input, to ensure good coating is melted and the melt temperature too high, so that the tendency of cracks to a minimum, to achieve control of the coating cracks, as show in figure 2.

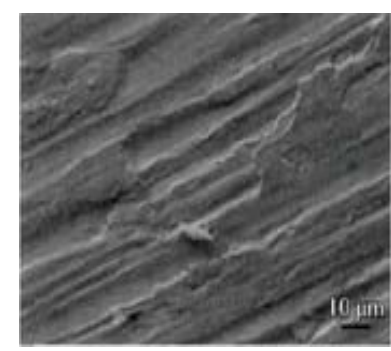

(a) Laser cladding layer

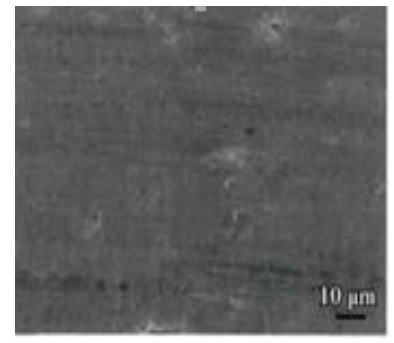

(b) Electron beam cladding layer

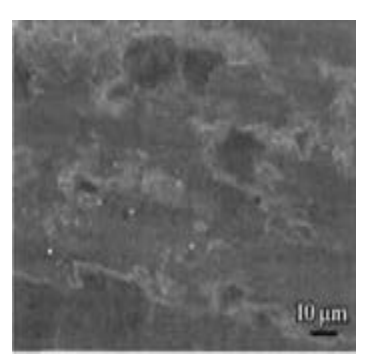

(c) Substrate material

Fig. 2: Worn morphologies of the substrate and the two surface composite layers synthesized

\section{Research Evolution of the Surface alloying by Intense pulsed electron beam}

In recent years, the technology of surface alloying by intense pulsed electron beam has been developed rapidly [15-16]. Intense pulsed electron beam can be used as a surface alloying by 
electron beam of generator. Under vacuum conditions, intense pulsed electron beam surface treatment performed. Electron beam of high energy density momentary action to the surface so that the temperature rises rapidly, while relying on the good thermal conductivity to achieve rapid cooling of the metal base, so that rapid melting of the coating layer is formed modifying layer, so that the treated surface is difficult to realize a conventional method to obtain the surface-modifying properties.

Intense pulsed electron beam is a new type of high-energy charged particle beam density, which accelerates electrons to an energy carrier, and has the following advantages: 1) high current pulsed electron beam at the substrate surface will produce a shock wave and shock and vibration effect; 2) accelerating voltage is the same, the incident energy of the electron beam scope far beyond the ion beam is conducive to the formation of a thick layer of modified; 3) high current pulsed electron beam surface treatment as the heat source energy absorption does not depend on the material surface optical properties, making energy efficiency is improved; 4) electron beam irradiation treatment to achieve high vacuum environment, improve the quality of the cladding, the cladding layer to reduce porosity and other microscopic defects and improve its anti-cracking ability[16].

Keming Zhang [17] conducted a surface alloying by intense pulsed electron beam for stainless steel. He discovered that after several times bombardment by electron beam, the sputtering of Bombardment make the layer of powder becomes thinner and thinner, and form Alloy layer and the diffusion layer in the stainless steel surface of rich titanium. They found that the surface layer is made of titanium mixed and phases constituting the phase and the content of -phase with the increase in the number of bombardment improves.

Tiejun Zhao [18] tries to make the surface alloying for Magnesium metal by intense pulsed electron beam. Samples corrosion resistance can be significantly improved, After the surface alloying for Magnesium metal with Aluminum. Surface alloying primarily affects the process of anodic dissolution, due to aluminum infiltration, so the dissolution rate decreased $\mathrm{Mg}$, dense oxide film formed on the surface, matrix isolation resulting in reduction of the corrosion rate of magnesium.

\section{Conclusions}

The technology of Surface alloying by electron beam as a new technology developed in recent years, it is relative to other methods of surface modification has unique advantages, , can be applied to almost all metal materials, making the surface modification of materials a powerful instrument. The technology of surface alloying by electron beam low cost, energy density, so that the material can be obtained excellent surface properties, in the future development of surface modification will become a hot research field.

\section{Acknowledgements}

This work was financially supported by the Foundation Project of the Guangxi Key Laboratory of Manufacturing system and Advanced Manufacturing Technology (14-045-15-011Z), and Education Project of Guangxi Zhuang Autonomous Region (LX2014466).

\section{References}

[1] Miao Gen, .Yao Shou-shao \& Zhang Shao-zong. Modern Surface Technology, China Machine Press, 2002.

[2] Wu Jian-hui \& Zhang Chuan-fu. High-energy beams technology and its application in corrosion and prevention. Corrosion Science and Protection Technology,14(3),pp.105,2002.

[3] Song Ren-guo, Chen Guang-nan \& Zhang Kun. Application and development of surface strengthening processes with laser and electron beams. Physics,29 (7),pp.411. 2000

[4] V.P. Rotshtein Yu.F \& Ivanov A.B. Markov Surface alloying of stainless steel 316 with copper 
using pulsed electron-beam melting of film - substrate system. Surface \& Coatings Technology,200,pp.6378-6383,2006.

[5] J.M. Poate, G. Foti \& D.S. Jacobson (Eds.), Surface Modification and Alloying by Laser, Ion, and Electron Beams, Plenum Press, United States of America, 1983.

[6] Dutta Majumdar J,Weisheit A, Mordike B L, etal. Laser surface alloying of Ti with Si,Al and $\mathrm{Si}+\mathrm{Al}$ for an improved oxidation resistance. Materials Science and Engineering A,l.266 (3), pp.123-124,1999.

[7] Mao Rong-shan: Study on Electron Beam Alloying Process and Properties of 40Cr Surface. MS., Chongqing University of Technology, China, 2013.

[8] Huang Yi-ping, Zhong Song \& Yao Hong-bo, The simulation of the thermal stress with Surface alloying by electron beam. Equipment Manufacturing Technology,1, pp.10,2014.

[9] Zhang Xiao-xia. Numerical Simulation for Surface Modification of Titanium by High Current Pulsed Electron Beam. MS., Dalian University of Technology, 2013.

[10] Song Wu-lin, Zhu Pei-di \& Huang Wei, study on vacuum laser cladding. Laser Technology. 21(4)pp.243.1997.

[11] Lu Bin-feng, Tang Pu-hong \& Lu Feng-gui, Comparison of Microstructure and Wear Resistance Composite Layers Synthesized by Laser Scanning and Electron Beam Scanning. China Surface Engineering.27 (4),pp.76,2014.

[12] Hamatani H \& Miyazaki Y. Optimization of an electron beam remelting of HVOF sprayed alloys and carbides. Surface and Coatings Technology.154,pp.176, 2002.

[13] Zenker R, Sacher D \& Buchwalder A. Hybrid technology hard coating-electron beam surface hardening. Surface and Coatings Technology,202, pp.804, 2002.

[14] He Jun, Zhang Bin-gang \& Zheng Kun. Cracking control of Surface alloying by electron beam. Transactions of The China Welding Institution,33pp.109, 2012.

[15] V.P. Rotshtein, D.I. Proskurovsky \& G.E.Ozur. Surface modification and alloying of metallic materials with low-energy high-current electron beams. Surface and Coating Technology, 180,pp.377,2004.

[16] Hao Sheng-zhi, Wu Ping-sheng \& Zhang Xiang-dong. Surface modification by high current pulsed electron beam. Heat Treatment of Metals,33(1),p.77,2008.

[17] Zhang Ke-min, Zou Jian-xin \& Yang Da-zhi, Surface alloying by electron beam with Ti alloy and corrosion resistance. Transactions of Materials and Heat Treatment.l.27(5) pp.108,2006.

[18] Zhao Tie-jun, Gao Bo \& Tian Xiao-mei. Modification and alloying of high purity magnesium surface with high current pulsed electron beam. Chinese Journal of Vacuum Science and Technology,28(1)pp.11,2008. 